\title{
Curvature and Geometry of Tessellating Plane Graphs
}

\author{
O. Baues ${ }^{1}$ and N. Peyerimhoff ${ }^{2}$ \\ ${ }^{1}$ Departement Mathematik, ETH-Zentrum, \\ CH-8092 Zürich, Switzerland \\ oliver@math.ethz.ch \\ ${ }^{2}$ Fakultät für Mathematik, Ruhr-Universität Bochum, \\ D-44780 Bochum, Germany \\ peyerim@math.ruhr-uni-bochum.de
}

\begin{abstract}
We show that the growth of plane tessellations and their edge graphs may be controlled from below by upper bounds for the combinatorial curvature. Under the assumption that every geodesic path may be extended to infinity we provide explicit estimates of the growth rate and isoperimetric constant of distance balls in negatively curved tessellations. We show that the assumption about geodesics holds for all tessellations with at least $p$ faces meeting in each vertex and at least $q$ edges bounding each face, where $(p, q) \in\{(3,6),(4,4),(6,3)\}$.
\end{abstract}

\section{Introduction}

The edge graph $\mathcal{G}$ of a tessellation or tiling of the plane may be viewed as an embedding of a graph which satisfies certain additional hypotheses. The most well known examples are given by tessellations of the Euclidean plane by regular triangles, quadrilaterals and hexagons. Other examples arise from hyperbolic geometry (see [Ro] for many beautiful examples). Obviously, these graphs inherit many geometric properties of the underlying plane geometry, and these properties are also reflected in the combinatorial structure of the tiling.

We introduce some notation and the definition of combinatorial curvature. For the vertex $v \in \mathcal{G}$, let $|v|$ denote the number of edges emanating from $v$, and, for a face $f$ of the tiling, let $E_{\partial f}$ denote the number of edges of $\mathcal{G}$ bounding $f$. Some information about the local combinatorial structure of the tiling is encoded in the characteristic function $\kappa$ which is defined on the set of vertices of $\mathcal{G}$, as follows:

$$
\kappa(v)=1-\frac{|v|}{2}+\sum_{f: v \in \partial f} \frac{1}{E_{\partial f}} .
$$


The function $\kappa$ may be viewed as a discrete analogue of curvature. (Compare, in particular, the discussion in Section 1 of this article.)

The main concern of this article is the influence of the combinatorial curvature function $\kappa$ on the geometry of $\mathcal{G}$. We investigate how upper curvature bounds determine properties of geodesics and volume growth in $\mathcal{G}$. It turns out that, as in Riemannian geometry, an important requirement for estimates of volume growth of distance balls is the emptiness of the cut locus. (This means that all distance minimizing geodesics in $\mathcal{G}$ may be extended to infinity.) We obtain the following result:

Theorem. Let $\mathcal{G}$ be a tessellating plane graph without cut locus. Let $-c<0$ be an upper bound for $\kappa$ on $\mathcal{G}$, and let $B_{r} \subset \mathcal{V}$ be a distance ball of radius $r$. Then

$$
\left|B_{r}\right| \geq(1+2 c)^{r}
$$

The theorem follows as a corollary to an estimate of the isoperimetric constant for distance balls in a negatively curved tessellating plane graph without cut locus. It was proved previously that a tessellating plane graph with $\kappa$ negative satisfies a strong isoperimetric inequality by Woess [Wo] and Żuk [Żu]. Earlier results for some particular classes of negatively curved graphs were obtained by Dodziuk [Do] and Soardi [So].

The main class of tessellating graphs we consider are tessellating graphs of type $(p, q)$, where $p, q$ are natural numbers which satisfy $1 / p+1 / q=\frac{1}{2}$. These graphs have the property that at least $p$ edges are emanating from each vertex, and at least $q$ edges are bounding each face of the tiling. Therefore tessellating graphs of type $(p, q)$ may be viewed as non-positively curved deformations of the regular tilings of the Euclidean plane, and we show that such graphs have empty cut locus:

Theorem. Let $\mathcal{G}$ be a tessellating plane graph of type $(p, q)$. Then the cut locus of $\mathcal{G}$ is empty, i.e., every geodesic ray in $\mathcal{G}$ may be extended to infinity.

We start our paper in Section 1 with some preliminary remarks on tilings of surfaces, the definition of the combinatorial curvature $\kappa$, and the discrete analogue of the GaußBonnet theorem. Also we introduce the characteristic function $\chi$, called the Eulercharacteristic, which is dual to $\kappa$, and which is defined on the set of faces of the tiling. For technical reasons, we chose to formulate most of our results in the paper in terms of the dual function $\chi$ which is just the curvature for the dual graph of $\mathcal{G}$.

In Section 2 we provide some preparatory material about polygons in tessellating graphs. In what follows, we crucially need properties of distance balls and spheres in a tessellating plane graph without cut locus. These properties are developed in Section 3.

In Section 4 we study the geometry of tessellating graphs of type $(p, q)$ more closely, and in particular we prove that the cut locus is empty in such graphs. The proof requires careful analysis of the boundary of distance balls.

In Section 5 we proof the isoperimetric inequality for distance balls in a tessellating plane graph without cut locus, where $\kappa$ is negative and bounded away from zero.

To round up the discussion, we consider in Section 6 regular tessellating graphs, i.e., tessellations where all vertices are of the same degree $n$. Corollary 6.4 exhibits 
a particular simple relation between Euler-characteristic and growth coefficients for $n \in\{3,4,6\}$. This translates into a corresponding result about curvature and growth coefficients in face-regular tilings. These are tilings where all faces have the same number of edges.

Our interest in the subject arose from several sources. For example, in the theory of tilings one studies tilings normal relative to a geometry, meaning that the faces are uniformly bounded in a chosen geometry on the plane, see [GS] for the Euclidean case. One can then ask which topological tilings may be realized as normal tilings, for example, of the Euclidean or hyperbolic plane. This and the question of enumeration of tilings were studied in an equivariant setting by Dress and Huson, see [DH]. It follows from Section 3.5 of [GS] that the curvature function $\kappa$ vanishes on $\mathcal{G}$ for a normal regular tiling of the Euclidean plane. Also, for tilings there exist various questions of type. See [Bo] for this in the context of random walks on graphs and circle packings.

The following question is in a similar spirit. Is a tessellating plane graph with (asymptotically) negative upper curvature bound also a Gromov-hyperbolic space, i.e., is it negatively curved in the sense of Gromov? Some affirmative answers were given by Żuk [ $\dot{\mathrm{Z}} \mathrm{u}$, Section 5], in particular for tessellations with at least seven faces meeting in each vertex and with a uniform bound on the number of edges of each face.

There is also a connection to (geometric) group theory which is already apparent from the techniques used to obtain the results of this paper. It is known (see [GHV]) that the Cayley graph of a finitely generated group $G$ is Gromov-hyperbolic if and only if $G$ satisfies a linear isoperimetric inequality. This condition is expressed in terms of a linear isoperimetric relation for plane diagrams which represent words which are trivial in $G$. These diagrams, on the other hand, may be viewed as parts of plane tilings.

\section{Tessellating Graphs}

Let $S$ be an orientable topological surface. We consider a connected simplicial graph

$$
\mathcal{G}=(\mathcal{V}, \mathcal{E})
$$

with the sets $\mathcal{V}, \mathcal{E}$ of vertices and edges, and an embedding of $\mathcal{G}$ in $S$. An embedding of $\mathcal{G}$ in $S$ is a continuous one-to-one map from a topological representation of $\mathcal{G}$ into $S$. For our purpose, we then identify $\mathcal{G}$ with its image in $S$. That $\mathcal{G}$ is simplicial means that the vertex set $\mathcal{V}$ is countable and that $\mathcal{G}$ has no loops nor multiple edges joining two vertices. Let $\mathcal{F}$ denote the family of connected components of the complement of the image of $\mathcal{G}$ in $S$. The elements of $\mathcal{F}$ are called the faces of $\mathcal{G}$. A face $f \in \mathcal{F}$ is called a polygon if $\bar{f}$ is homeomorphic to a closed disk and if its boundary is a closed path of edges without repeated vertices. The edges of $\mathcal{G}$ which are contained in the boundary cycle $\partial f$ of a polygon $f \in \mathcal{F}$ are then called the sides of $f$. If the polygon $f$ has $k$ sides it is called a $k$-gon. The number of edges emanating from a vertex $v \in \mathcal{V}$ is called the degree of $v$, denoted by $|v|$. (For the basic notions of graph theory and surface embeddings of graphs refer to $[\mathrm{Wh}]$ and $[\mathrm{Yo}]$.) 
Definition 1.1. An embedded graph $\mathcal{G}$ in $S$ is called tessellating, if the following conditions are satisfied:

(i) Any edge is a side of precisely two different faces.

(ii) Any two faces are disjoint or have precisely either a vertex or a side in common.

(iii) Any face $f \in \mathcal{F}$ is a polygon with finitely many sides.

(iv) Every vertex has finite degree.

In the special case $S=\mathbf{R}^{2}$ we call $\mathcal{G}$ a tessellating plane graph. We only consider locally finite graphs in this article. A graph is called locally finite if, for every $p \in S$, there is an open set containing $p$ which meets only finitely many faces of $\mathcal{G}$.

Remark. Condition (ii) is a customary convexity assumption for a tiling (see Section 3.2 of $[\mathrm{GS}]$, or $[\mathrm{DH}])$ which seems to be rather strong. It can be relaxed to:

(ii)* Any two faces have at most one side in common.

However, in most of the cases we are interested in, condition (ii)* already implies condition (ii). For example, the reader should convince himself that this is true for tessellating graphs of type $(p, q)$. (See Section 4 and in particular Lemma 4.2.)

Since each face of a tessellating graph is required to be an open disk, the embedding of the edge graph $\mathcal{G}$ is a 2-cell embedding. The importance of the fact that each face of a tessellation is a 2-cell stems from Euler's formula which is used frequently in this paper. We state it here as follows: let $\mathcal{G}$ be a finite 2-cell embedded graph in a closed surface $S_{g}$ of genus $g$, and let $p, q, r$ be the number of vertices, edges and faces of $\mathcal{G} \subset S_{g}$, then

$$
p-q+r=2-2 g \text {. }
$$

For a 2-cell embedded graph $\mathcal{G}$, let $\mathcal{G}^{*}$ denote its (geometric) dual which is also a connected embedded graph. We remark that if $\mathcal{G}^{*}$ is simplicial it is also 2 -cell embedded. If $\mathcal{G}$ is tessellating, then (i) and (ii) of the definition above imply that $\mathcal{G}^{*}$ is simplicial. Also, since $\mathcal{G}$ is simplicial, $\mathcal{G}^{*}$ satisfies (i). Condition (ii) for $\mathcal{G}$ implies that condition (ii) also holds for $\mathcal{G}^{*}$. The fact that the boundaries of the faces of $\mathcal{G}$ are simple cycles implies that the same is true for $\mathcal{G}^{*}$. Conditions (iii) and (iv) are then dual to each other, so that $\mathcal{G}^{*}$ is also a tessellating graph.

Definition 1.2. Let $f \in \mathcal{F}$ be a $k$-gon of the tessellating graph $\mathcal{G}$ in the surface $S$. The Euler-characteristic of $f$ is defined as the rational number

$$
\chi(f):=1-\frac{k}{2}+\sum_{v \in \partial f} \frac{1}{|v|} .
$$

The Euler-characteristic of finitely many faces $A \subset \mathcal{F}$ is then defined as

$$
\chi(A):=\sum_{f \in A} \chi(f) .
$$


Remark. In $[\dot{Z} \mathrm{u}]$ a face of a tiling is called a hyperbolic polygon if its Euler-characteristic is $<0$.

Let $\mathcal{G}$ be a 2-cell embedded tessellating graph in the closed surface $S_{g}$ and let $\chi\left(S_{g}\right)=$ $2-2 g$ be the Euler-characteristic of $S_{g}$. Using Euler's formula (1), as well as properties (i) and (iii) of a tessellating graph, it is easy to see that the following identity holds:

$$
\chi(\mathcal{F})=\chi\left(S_{g}\right)
$$

That is, the sum over the Euler-characteristics of the polygons of a tessellating graph in $S_{g}$ equals the Euler-characteristic of the cell decomposition induced by the tessellation.

Now let $v \in \mathcal{V}$ be a vertex of the tessellating graph $\mathcal{G}$, let $\mathcal{G}^{*}$ be the dual graph and let $f(v)$ be the face in $\mathcal{G}^{*}$ corresponding to $v$. The combinatorial analogue of curvature in $v$ may be defined as follows:

Definition 1.3. The curvature $\bar{\kappa}(v)$ of $\mathcal{G}$ in $v$ is defined by the expression

$$
\bar{\kappa}(v):=2 \pi \chi(f(v)) .
$$

The total curvature $\bar{\kappa}(V)$ of finitely many vertices $V \subset \mathcal{V}$ is

$$
\bar{\kappa}(V):=\sum_{v \in V} \bar{\kappa}(v) .
$$

Remark. Note that $\bar{\kappa}(v)=2 \pi \kappa(v)$, where $\kappa$ is the combinatorial curvature function which is used in the Introduction.

The following combinatorial version of the Gauß-Bonnet theorem follows from the summation formula (2):

Theorem 1.4 (Combinatorial Gauß-Bonnet Formula). Let $S$ be a closed oriented topological surface and let $\mathcal{G}=(\mathcal{V}, \mathcal{E})$ be a locally finite tessellating graph in $S$. Then

$$
\bar{\kappa}(\mathcal{V})=2 \pi \chi(S) .
$$

The theorem seems to be part of mathematical folklore and it is difficult to locate a reference. Thurston states the Gauß-Bonnet formula for Euclidean cone metrics on surfaces in [Thu]. Other combinatorial versions of Gauß-Bonnet can be found in [Pó], [GP, p. 200] or [BB].

Remark. The definition of curvature in a tessellating graph $\mathcal{G}$ may be interpreted geometrically as follows: The graph $\mathcal{G}$ determines a Euclidean cone metric on the surface $S$. In this metric, each $k$-gon of $\mathcal{G}$ is isometric to a regular $k$-gon in Euclidean space with side length 1 . It is locally Euclidean except, possibly, at the vertices of $\mathcal{G}$. The curvature in the vertex $v \in \mathcal{G}$ is then naturally defined as the $2 \pi$-angle-defect of the flat polygons 
meeting in $v$. If $r$ polygons with side-numbers $k_{1}, \ldots, k_{r}$ meet in the vertex $v$, one obtains

$$
\bar{\kappa}(v)=2 \pi-\sum_{i=1}^{r} \frac{k_{i}-2}{k_{i}} \pi=2 \pi-\theta .
$$

Hence a neighbourhood of $v$ is isometric to a cone of angle $\theta$ with apex $v$.

Remark. Cohn-Vossen proved the following result for complete non-compact Riemannian 2-manifolds $S$ : Assume the existence of $\lim _{j \rightarrow \infty} \int_{K_{j}} \kappa(q) d \operatorname{vol}(q)$ for all compact exhaustions $K_{j}$ of $S$, and denote it by $\int_{S} \kappa(q) d \operatorname{vol}(q)$. Then

$$
\int_{S} \kappa(q) d \operatorname{vol}(q) \leq 2 \pi \chi(S)
$$

Further investigations (e.g., [Li]) lead to relations between finite total curvature, finite topological type and quadratic volume growth of geodesic balls. It seems natural to expect corresponding combinatorial results.

\section{Polygons in Tessellating Plane Graphs}

For the sake of simplicity of the exposition, we consider, from now on, only tessellating plane graphs, although all notions are valid (mutatis mutandis) in the more general context of surfaces.

We give a few additional definitions and notations. Let $A \subset \mathcal{F}$ be a finite subset. We say $e \in \mathcal{E}$ belongs to $A$ if $e$ is a side of some $f \in A$, and similarly we say $v \in V$ belongs to $A$ if $v$ is the endpoint of some edge which belongs to $A$. $A$ is called a polygon, if

$$
\mathcal{P}(A)=\bigcup_{f \in A} \bar{f} \subset \mathbf{R}^{2}
$$

is homeomorphic to a closed disk. Then the (topological) boundary $\partial \mathcal{P}(A)$ is a closed path of edges without repeated vertices, and the boundary cycle of $A$ is defined as

$$
\partial A=\{e \in \mathcal{E} \mid e \subset \partial \mathcal{P}(A)\} .
$$

We write $E_{\partial A}$ for $|\partial A|$, and, for any subset $B$ of $\mathcal{F}, F_{B}=|B|$ denotes the number of faces, $E_{B}$ the number of edges, and $V_{B}$ the number of vertices of $\mathcal{G}$ which belong to $B$. We say that a vertex $v$ is an interior vertex of $A$ if it belongs to a face of $A$ but not to an edge of $\partial A$, and we write

$$
v \in \stackrel{\circ}{A}
$$

to denote that $v$ is an interior vertex of $A$. Also, by abuse of notation, we write $v \in \partial A$, if $v$ belongs to an edge which is contained in $\partial A$.

If $A \subset \mathcal{F}$ is a polygon and $v$ belongs to $\partial A$, then the inner degree $|v|_{A}^{\mathrm{i}}$ of $v$ (relative to $A$ ) is the number of faces $f \in A$ meeting in $v$, and the exterior degree $|v|_{A}^{\mathrm{e}}$ is $|v|_{A}^{\mathrm{e}}:=|v|-|v|_{A}^{\mathrm{i}}$. If it is clear from the context which polygon is referred to, we will 
just write $|v|^{\mathrm{i}}$ for $|v|_{A}^{\mathrm{i}}$, and $|v|^{\mathrm{e}}$ for $|v|_{A}^{\mathrm{e}}$. The Euler-characteristic $\chi(A)$ can be computed by knowing only the inner and exterior degrees of the boundary vertices of $A$ :

Proposition 2.1. Let $A \subset \mathcal{F}$ be a polygon of a tessellating plane graph $\mathcal{G}$. Then

$$
\chi(A)=1-\frac{E_{\partial A}}{2}+\sum_{v \in \partial A} \frac{|v|^{\mathrm{i}}}{|v|} .
$$

Proof. We have

$$
\chi(A)=\sum_{f \in A} \chi(f)=\sum_{f \in A}\left(1-\frac{E_{\partial f}}{2}+\sum_{v \in \partial f} \frac{1}{|v|}\right)=F_{A}-E_{A}+\frac{E_{\partial A}}{2}+\sum_{f \in A} \sum_{v \in \partial f} \frac{1}{|v|} .
$$

Since $A$ is a polygon, we have from Euler's formula that

$$
F_{A}-E_{A}+V_{A}=1
$$

and also

$$
\sum_{f \in A} \sum_{v \in \partial f} \frac{1}{|v|}=V_{A}-\sum_{v \in \partial A} \frac{|v|^{\mathrm{e}}}{|v|} .
$$

The latter formula holds since, by condition (iii), in a tessellating graph the degree of a vertex $v$ coincides with the number of different faces meeting in $v$. Using $V_{\partial A}=E_{\partial A}$ it follows from (5) and (6) that

$$
\chi(A)=1+\frac{E_{\partial A}}{2}-V_{A}+\sum_{f \in A} \sum_{v \in \partial f} \frac{1}{|v|}=1-\frac{E_{\partial A}}{2}+\sum_{v \in \partial A} \frac{|v|^{\mathrm{i}}}{|v|} .
$$

The following formula is due to Lyndon (see Corollary 2.2 of [Ly]) and is used in the next section. For the convenience of the reader, we give a proof in our terminology.

Theorem 2.2 (Lyndon). Let $\mathcal{G}$ be a tessellating plane graph, A a polygon, and $1 / p+$ $1 / q=\frac{1}{2}$. Assume that $|v| \geq p$ for all interior vertices of $A$, and that $E_{\partial f} \geq q$ for all $f \in A$. Then we have

$$
\sum_{v \in \partial A}\left(\frac{p}{2}-|v|^{\mathrm{i}}\right) \geq p
$$

Proof. Let $p \neq 0$ be an arbitrary real number, and let $A$ be a polygon of $\mathcal{G}$. We conclude

$$
\begin{aligned}
\chi(A) & =\sum_{f \in A}\left(1-\frac{E_{\partial f}}{2}+\sum_{v \in \partial f} \frac{1}{|v|}\right) \\
& =\sum_{f \in A}\left(1+\sum_{v \in \partial f}\left(\frac{1}{|v|}-\frac{1}{p}\right)+\left(\frac{1}{p}-\frac{1}{2}\right) E_{\partial f}\right) \\
& =\sum_{f \in A}\left(1+\left(\frac{1}{p}-\frac{1}{2}\right) E_{\partial f}\right)+\sum_{v \in A}\left(1-\frac{|v|}{p}\right)+\sum_{v \in \partial A}\left(\frac{|v|^{\mathrm{i}}}{|v|}-\frac{|v|^{\mathrm{i}}}{p}\right) .
\end{aligned}
$$


On the other hand, we obtain with (4)

$$
\chi(A)=1-\frac{E_{\partial A}}{2}+\sum_{v \in \partial A} \frac{|v|^{\mathrm{i}}}{|v|}=1+\sum_{v \in \partial A}\left(\frac{|v|^{\mathrm{i}}}{|v|}-\frac{1}{2}\right) .
$$

Combining both equations yields

$$
1=\sum_{v \in \partial A}\left(\frac{1}{2}-\frac{|v|^{\mathrm{i}}}{p}\right)+\sum_{f \in A}\left(1+\left(\frac{1}{p}-\frac{1}{2}\right) E_{\partial f}\right)+\sum_{\substack{\mathrm{o} \\ v \in A}}\left(1-\frac{|v|}{p}\right) .
$$

Note that, in the case $1 / p+1 / q=\frac{1}{2}$, we have $\left(1 / p-\frac{1}{2}\right) E_{\partial f} \leq-1$ and $1-|v| / p \leq 0$. Consequently, the last two terms on the right-hand side of (7) are $\leq 0$, which implies Lyndon's inequality.

\section{Tessellating Graphs without Cut Locus}

The distance $d\left(f, f^{\prime}\right)$ of two faces $f, f^{\prime}$ of a tessellating graph $\mathcal{G}=(\mathcal{V}, \mathcal{E}, \mathcal{F})$ is the combinatorial distance of their corresponding vertices in the dual $\mathcal{G}^{*}$. Two faces are called neighbours if they have an edge in common. A sequence $f_{1}, f_{2}, \ldots$ of faces forms a connected path if any two subsequent faces $f_{j}, f_{j+1}$ are neighbours. Such a path corresponds to a connected path of edges in the dual $\mathcal{G}^{*}$. A (minimal) geodesic in $\mathcal{G}$ is then a path of faces $f_{1}, f_{2}, \ldots$ satisfying $d\left(f_{i}, f_{j}\right)=|i-j|$ for all $i, j$.

Definition 3.1. Let $f_{0} \in \mathcal{F}$ be a fixed face and let $d_{0}(f):=d\left(f_{0}, f\right)$, for all $f \in \mathcal{F}$, be the distance function of $f_{0}$. The cut locus $C\left(f_{0}\right)$ of $f_{0}$ is the set

$$
C\left(f_{0}\right):=\left\{f^{\prime} \in \mathcal{F} \mid d_{0}(f) \leq d_{0}\left(f^{\prime}\right) \text { for all neighbours } f \text { of } f^{\prime}\right\} .
$$

That is, $C\left(f_{0}\right) \subset \mathcal{F}$ is the set where the function $d_{0}$ attains its local maxima.

We associate to the face $f_{0} \in \mathcal{F}$ the distance spheres and distance balls:

$$
\begin{aligned}
& A_{r}=\left\{f \in \mathcal{F} \mid d_{0}(f)=r\right\}, \\
& B_{r}=\left\{f \in \mathcal{F} \mid d_{0}(f) \leq r\right\} .
\end{aligned}
$$

Our aim is to show

Theorem 3.2. If $\mathcal{G}$ is a tessellating plane graph without cut locus, then all distance balls $B_{r}$ are polygons. Moreover, the boundary cycle $\partial B_{r}$ imposes a (cyclic) enumeration of $A_{r+1}=\left\{f_{1}, \ldots, f_{k}\right\}$ such that precisely subsequent faces intersect, and each intersection contains a vertex $v \in \partial B_{r}$.

First we analyze the consequences of the global condition $C\left(f_{0}\right)=\emptyset$ on the neighbourhoods of vertices in distance spheres. For $v \in \mathcal{V}$ let

$$
S(v)=\{f \in \mathcal{F} \mid v \text { is a vertex of } f\}
$$


be the star of $v$ and $A_{r}(v)=A_{r} \cap S(v)$. Let $U \subset \mathbf{R}^{2}$ be a small neighbourhood of $v$. We call the intersections of $U$ with the closures of faces $f \in S(v)$ the segments of $U$.

Proposition 3.3. If $C\left(f_{0}\right)=\emptyset$, then $U-\bigcup_{f \in A_{r}(v)} \bar{f}$ has at most two connected components. The faces of the segments in one of these components all belong either to $\bigcup_{l>r} A_{l}(v)$ or to $\bigcup_{l<r} A_{l}(v)$.

Proof. Since $\left|d_{0}(f)-d_{0}\left(f^{\prime}\right)\right| \leq 1$ for neighbouring faces $f, f^{\prime}$, we can conclude from $d_{0}(f)>r$ for one of the faces in a component of $U-\bigcup_{f \in A_{r}(v)} \bar{f}$ that the same is true for all faces in this component. We assume now that $U-\bigcup_{f \in A_{r}(v)} \bar{f}$ has at least three components, and that two of them contain faces $f_{1}$ and $f_{2}$, respectively, satisfying $d_{0}\left(f_{i}\right)<r$. We still use paths of faces in $\mathcal{G}$, but we consider them here as paths in the dual graph $\mathcal{G}^{*}$. In this sense, we choose geodesics $c_{i}$ joining $f_{i}$ and $f_{0}$. There are two ways to join the $f_{i}$ along the boundary cycle of $v$. Any of these two paths can be combined with (parts of) the $c_{i}$ to form a simple closed path $c$. We choose the path such that $v \in \partial B$, where $B$ is the polygon containing all faces of the bounded part of $\mathbf{R}^{2}-c$ as well as the faces which belong to $c$. Note that $\partial B$ belongs only to faces of $c_{1}$ or $c_{2}$. On the other hand, $B$ contains a face $f$ with $d_{0}(f)=r$ on the boundary cycle of $v$. Hence, since $B$ is finite, there is a face $f^{\prime} \in B$ with maximal distance $d_{0}\left(f^{\prime}\right) \geq r$. This face is in $C\left(f_{0}\right)$ since all it neighbours are contained in $B$.

In the case that there are two components of $U-\bigcup_{f \in A_{r}(v)} \bar{f}$ with faces $f$ satisfying $d_{0}(f)>r$, we take two faces $f_{1}$ and $f_{2}$ in $A_{r}(v)$ which separate these components, and repeat the previous argument.

Proposition 3.4. If $C\left(f_{0}\right)=\emptyset$, then $\left|A_{r}(v)\right| \leq 2$.

Proof. Let $f_{1}, f_{2}$ and $f_{3}$ be three faces in $A_{r}(v)$. We choose geodesics $c_{1}, c_{2}$ from $f_{1}, f_{2}$ to $f_{0}$. As in the previous proof, we connect (part of) the $c_{i}$ along the boundary cycle of $v$ to form a simple closed path $c$. Moreover, we can assume that we chose $f_{1}, f_{2}$ and $f_{3}$ in such a way that $f_{3}$ is on the boundary cycle part of $c$. As in the previous proof, this gives us a polygon $B$ with $v \in \partial B$. Again, there is a face $f^{\prime} \in B$ with maximal distance to $f_{0}$ such that all its neighbours in $\mathcal{G}$ are contained in $B$. Consequently, $f^{\prime} \in C\left(f_{0}\right)$.

Proof of the First Part of Theorem 3.2. Let $B=B_{r}$ be a distance ball of $f_{0}$. Any edge $e \in \partial B$ is a side of two faces $f, f^{\prime}$ which belong to $A_{r}$ and $A_{r+1}$, respectively. We show first that, at every vertex $v \in \partial B$, there meet precisely two edges of $\partial B$. This implies that $\partial B$ is a union of simple cycles. From Proposition 3.4, we know that $U-$ $\bigcup_{f \in A_{r}(v)} \bar{f}$ contains exactly one connected component whose segments belong to faces in $\bigcup_{l>r} A_{l}(v)$. Then the edges of $\partial B$ meeting at $v$ coincide with the two boundary edges of this component. Thus, we proved that the connected components of $\partial B$ are simple cycles.

Since $\mathcal{B}=\bigcup_{f \in B} \bar{f}$ is a compact connected set, there is a cycle $c_{0}$ of $\partial B$ enclosing $\mathcal{B}$. Therefore, all other possible boundary cycles are contained in the bounded component enclosed by $c_{0}$. However, the existence of such cycles contradicts the assumption $C\left(f_{0}\right)=\emptyset$. 
Proposition 3.5. If $C\left(f_{0}\right)=\emptyset$, then, for every $f \in A_{r+1}$, the intersection $\partial f \cap \partial B_{r}$ is a connected path of edges.

Proof. Clearly, one component of the (topological) boundaries $\partial \bar{f} \cap \partial \bar{B}_{r}$ is a connected path containing at least one edge. Assume that there is another component. Since $\partial B_{r}$ is a cycle, the two components may be connected along a path $w_{1} \subset \partial B_{r}$ such that $w_{1}$ encloses, together with a part of $\partial f$, a compact area which contains cut locus.

Proof of the Second Part of Theorem 3.2. By the previous proposition, the sequence of connected paths $\partial f \cap \partial B_{r}$, for $f \in A_{r+1}$, along the boundary cycle $\partial B_{r}$ gives an enumeration of $A_{r+1}$. Clearly, two faces with subsequent indices intersect in at least one vertex $v \in \partial B_{r}$. Suppose $f, \tilde{f}$ are not subsequent along $\partial B_{r}$, then the same argument as in the previous proof shows that they cannot intersect.

\section{Distance Balls in Tessellating Graphs of Type $(p, q)$}

A well known fact in Riemannian geometry is that complete, simply connected manifolds with non-positive curvature have empty cut locus. This naturally leads to the assumption that the same could be true for tessellating plane graphs where all faces have non-positive Euler-characteristic. The answer to this question is not known to us in full generality. Here, we can prove the emptiness of the cut locus for some natural classes of nonpositively curved graphs.

A tessellating graph $\mathcal{G}$ is called of type $(p, q)$ if, for all $v \in \mathcal{V}, f \in \mathcal{F}$,

$$
|v| \geq p \quad \text { and } \quad E_{\partial f} \geq q,
$$

for natural numbers $p, q$ with $(p, q) \in\{(3,6),(4,4),(6,3)\}$, or equivalently which satisfy $1 / p+1 / q=\frac{1}{2}$. It is clear that $\chi \leq 0$ for a tessellating graph of type $(p, q)$. We are going to prove the following result:

Theorem 4.1. Let $\mathcal{G}$ be a plane tessellating graph of type $(p, q)$. Then we have $C\left(f_{0}\right)=$ $\emptyset$ for every face $f_{0} \in \mathcal{F}$.

Before we present the proof we introduce some useful notions. Let $B$ be a polygon. We label the boundary vertices of $B$ as follows: $v \in \partial B$ obtains the label $a$ if $|v|_{B}^{\mathrm{i}}=1$, in the case $q \in\{6,4\}$, and if $|v|_{B}^{\mathrm{i}} \leq 3$, in the case $q=3$. Otherwise, $v$ obtains the label $b$. We call $B$ admissible if the label sequence along the closed path $\partial B$ has the property that every label $b$ is enclosed by vertices of label $a$ which both satisfy $|v|_{B}^{\mathrm{i}}=1$.

Now let $B$ and $P$ be polygons of the graph $\mathcal{G}$, and $\partial B, \partial P \subset \mathcal{E}$ their boundary cycles. Assume that $B, P$ satisfy the following properties:

(i) $\partial P \cap \partial B \neq \emptyset$,

(ii) $\partial P \cap \partial B$ is a connected path (of edges),

(iii) $P \cap B=\emptyset$. 

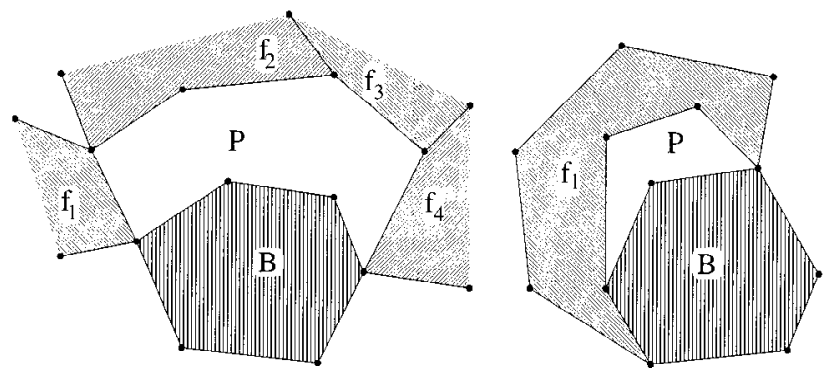

Fig. 1. Examples of bridges of length 4 and 1 . The second bridge is attached to $B$ at the left side.

Note that in particular $\partial P$ and $\partial B$ have at least one edge in common. If $B, P$ satisfy (i)-(iii), the set

$$
\Gamma=\{f \in \mathcal{F}-P \mid \text { an edge of } f \text { belongs to } \partial P-\partial B\}
$$

is called a bridge over $B$ of length $L(\Gamma)=|\Gamma|$. Let $v_{1}, v_{2}$ denote the two endpoints of the path $\partial P \cap \partial B$. (Note that, since $P$ is assumed to be a polygon, always $v_{1} \neq v_{2}$.) We say that $\Gamma$ is attached at one side to $B$ if there exists $f \in \Gamma$ such that $\partial f \cap \partial B$ contains an edge emanating from $v_{1}$ or $v_{2}$. In particular, if there exists such an $f \in \Gamma$ for both endpoints, we say that $\Gamma$ is attached at both sides. Examples of bridges are given in Fig. 1.

Lemma 4.2 (Bridge Lemma). Let $\mathcal{G}$ be a graph of type $(p, q)$, and let $B, P$ be polygons of $\mathcal{G}$. Assume further that $B$ is admissible, and that $B, P$ satisfy the properties above. Then

$$
L(\Gamma) \geq q-2 .
$$

If, additionally, $\Gamma$ is attached at one side to $B$, then, in the case $q \in\{3,4\}$,

$$
L(\Gamma) \geq q-1 .
$$

If $\Gamma$ is attached at both sides to $B$, then, in the case $q=3$,

$$
L(\Gamma) \geq 3 .
$$

Proof. Let $w_{1}$ denote the connected path of edges in $\partial P$, disjoint to $\partial B$ (except for the endpoints $v_{1}, v_{2} \in \partial B$ ). Let $w_{2}$ be the complementary path along $\partial P \cap \partial B$, but without the edges containing $v_{1}, v_{2}$.

Since $\mathcal{G}$ satisfies the conditions of Lyndon's formula (Theorem 2.2), we can conclude

$$
\begin{aligned}
p & \leq \sum_{v \in \partial P}\left(\frac{p}{2}-|v|_{P}^{\mathrm{i}}\right) \\
& =\sum_{v \in w_{1}}\left(\frac{p}{2}-|v|_{P}^{\mathrm{i}}\right)+\sum_{v \in w_{2}}\left(\frac{p}{2}-|v|_{P}^{\mathrm{i}}\right) .
\end{aligned}
$$

Since $|v| \geq p$, for any $v \in \partial P$, it follows that the first sum is $\leq(L(\Gamma)+1)(p / 2-1)$, and also, since $B$ is admissible, that the second sum is $\leq p / 2-1$. So far, we proved $q-2 \leq L(\Gamma)$, for any bridge $\Gamma$. 
Assume now that $\Gamma$ is attached at $v_{1}$, and that $q \in\{3,4\}$. In the case that

$$
\sum_{v \in w_{2}}\left(\frac{p}{2}-|v|_{P}^{\mathrm{i}}\right)>0
$$

we have necessarily that $v_{1}$ and $v_{2}$ carry label $a$, and satisfy $\left|v_{1}\right|_{B}^{\mathrm{i}}=\left|v_{2}\right|_{B}^{\mathrm{i}}=1$. Since $\Gamma$ is attached at $v_{1}$, it follows that $\left|v_{1}\right|_{P}^{\mathrm{i}} \geq p-2 \geq p / 2$. Therefore the first sum in (8) is $\leq L(\Gamma)(p / 2-1)$, and hence $q-1 \leq L(\Gamma)$. If $\Gamma$ is attached at both sides we get $q \leq L(\Gamma)$, accordingly. So far, we proved the lemma under the assumption (9).

We assume now that

$$
\sum_{v \in w_{2}}\left(\frac{p}{2}-|v|_{P}^{\mathrm{i}}\right) \leq 0
$$

In this case it is always true that $q-1 \leq L(\Gamma)$. Therefore, we are left to prove that $3 \leq L(\Gamma)$, for a bridge $\Gamma$ which is attached at both sides, in a tessellating graph of type $(6,3)$, under the assumption (10). We assume first that $\sum_{v \in w_{2}}\left(3-|v|_{P}^{\mathrm{i}}\right)=0$. This is only possible if one of $v_{1}, v_{2}$ satisfies $|v|_{B}^{\mathrm{i}}=1$, or $v_{1}$ and $v_{2}$ are both of label $a$. In the first case, we get $3 \leq L(\Gamma)$, with the same reasoning as before. In the latter case, we deduce from $\left|v_{1}\right|_{B}^{\mathrm{i}},\left|v_{2}\right|_{B}^{\mathrm{i}} \leq 3$ that $\left|v_{1}\right|_{P}^{\mathrm{i}},\left|v_{2}\right|_{P}^{\mathrm{i}} \geq 2$ and we deduce from (8) that

$$
6 \leq 2+(L(\Gamma)-1) 2
$$

and from this $3 \leq L(\Gamma)$. Finally, we conclude from $\sum_{v \in w_{2}}\left(p / 2-|v|_{P}^{\mathrm{i}}\right)<0$ that

$$
6<(L(\Gamma)+1) 2,
$$

and hence the claim of the lemma always follows.

Now we are ready for the key step in the proof of Theorem 4.1:

Proposition 4.3. Let $\mathcal{G}$ be of type $(p, q)$, let $B$ be an admissible polygon and let $T=\{f \in \mathcal{F} \mid d(f, B) \leq 1\}$ be the distance 1 tube around $B$. Then $T$ is again an admissible polygon and, for all faces $f \in T-B, \partial f \cap \partial T$ is a connected path of edges of length $\geq 1$.

Proof. We remark first that for $f \in T-B, \partial f \cap \partial T$ is a connected path. If the intersection of the (topological) boundaries $\partial \bar{f} \cap \partial \bar{B}$ were not connected, there would be a bridge over $B$ of length 1 which is attached at one side. The Bridge Lemma (Lemma 4.2) implies that such a bridge is of length $\geq q-1 \geq 2$. Consequently, $\partial f \cap \partial B$ is a connected path of edges. The labeling of the vertices in $\partial B$ ensures that the length of this path is $\leq 2$.

Following the edges of the boundary cycle $\partial B$, we obtain an enumeration of the faces of $T-B$, i.e., $T-B=\left\{f_{1}, f_{2}, \ldots, f_{k}\right\}$. Any two faces $f_{i}, f_{j} \in T-B$ with $\bar{f}_{i} \cap \bar{f}_{j} \neq \varnothing$ have subsequent indices $(\bmod k)$ ). For otherwise, $f_{i}, f_{j}$ would define a bridge $\Gamma$ over $B$ of length 2 which is attached at both sides. This is impossible because of the Bridge Lemma.

We show now that each face $f \in T-B$ contributes an edge to $\partial T$. From what was said before, it is already clear that, in the case $q=6, \partial f$ contributes at least two edges to 
$\partial T$. We assume now $q \in\{3,4\}$. Let $v_{1}, v_{2}$ denote the two endpoints of the path $\partial f \cap \partial B$. First we consider the case where $|\partial f \cap \partial B|=1$. Since $B$ is admissible at least one of $v_{1}$, $v_{2}$, say $v_{1}$, carries the label $a$. Therefore, $\left|v_{1}\right|_{B}^{\mathrm{e}} \geq 3$, and it follows that $f$ does not share an edge with its corresponding neighbour $f_{1} \in T-B$ in $v_{1}$. Consequently, $f$ contributes at least one edge to $\partial T$, in the case $q=3$, and at least two edges, in the case $q=4$. Next, we have to consider the remaining case $|\partial f \cap \partial B|=2$. In this case, necessarily $\left|v_{1}\right|_{B}^{\mathrm{i}}=\left|v_{2}\right|_{B}^{\mathrm{i}}=1$, and hence $f$ does not share an edge with any one of its neighbours along $\partial T$. Again, $f$ contributes at least one edge to $\partial T$, in the case $q=3$, and at least two edges, in the case $q=4$.

The previous considerations imply, in particular, that $\partial T$ is a closed path without repeated vertices. Consequently, $T$ is a polygon. We have to show that $T$ is again admissible. In the cases $q=6, q=4$ each $f \in T-B$ contributes at least two edges to $\partial T$, and the claim is immediate. Assume now $q=3$. If $v \in \partial T$ is of label $b$ it satisfies $|v|_{T}^{\mathrm{i}}>3$. Then also $v \in \partial B$, and $|v|_{B}^{\mathrm{i}}>1$. Since $B$ is admissible, both neighbours of $v$ along $\partial B$ have label $a$, and consequently the two faces $f_{1}, f_{2} \in T-B$ which meet in $v$, do not share an edge with any neighbour along $\partial T$, and also satisfy $\left|\partial f_{1} \cap \partial B\right|=\left|\partial f_{2} \cap \partial B\right|=1$. Therefore $f_{1}, f_{2}$ contribute at least two edges to $\partial T$, and hence $T$ is admissible.

Proof of Theorem 4.1. We show, inductively, that, for all $r \in \mathbb{N}$, the distance balls $B_{r}\left(f_{0}\right)$ are admissible polygons, and that every face $f \in A_{r}\left(f_{0}\right)$ contributes a connected path of length $\geq 1$ to $\partial B_{r}\left(f_{0}\right)$. This obviously holds for $r=0$. The induction step is a particular case of Proposition 4.3. This finishes the proof of $C\left(f_{0}\right)=\emptyset$.

\section{Growth of Negatively Curved Tessellating Graphs}

Let $\mathcal{G}$ be a plane tessellating graph without cut locus. In the presence of negative curvature bounded from above, we have a linear isoperimetric inequality between the volume of distance spheres and the volume of balls. We show that the isoperimetric constant for distance balls depends linearly on the curvature bound.

With the notational conventions from Section 3 we define $a_{k}=\left|A_{k}\right|, b_{k}=\left|B_{k}\right|$. Then we have

Theorem 5.1 (Isoperimetric Inequality for Distance Balls). Let $\mathcal{G}$ be a tessellating plane graph without cut locus. Assume there exist constants $c>0$ and $d \in \mathbb{N} \cup\{\infty\}$ such that $\chi(f) \leq-c$, for all $f \in \mathcal{G}$, and $|v| \leq d$, for all $v \in \mathcal{V}$. Then we have, for all $k \geq 0$,

$$
a_{k+1} \geq \frac{d}{d-1} 2 c b_{k}
$$

where we define $d /(d-1)=1$ in the case $d=\infty$.

Remark. A related result was proved independently by Woess [Wo] and Żuk [Żu]. They showed that a strictly negative (asymptotic) curvature bound implies a strong isoperimetric inequality $\operatorname{area}(\partial A) \geq \varepsilon \cdot \operatorname{vol}(A)$, where $A$ is any polygon. However, in both references, the interest is focused on the existence of the constant $\varepsilon>0$. The 
dependence of $\varepsilon$ on upper curvature bounds is not discussed. Isoperimetric inequalities for some special families of negatively curved graphs were also given in [Do] and [So]. The notions of $\operatorname{area}(\partial A)$ and $\operatorname{vol}(A)$ used by these authors differ somewhat from ours. This makes no (qualitative) difference in the case of bounded geometry.

Obviously, the isoperimetric inequality implies exponential growth of distance balls.

Corollary 5.2. Let $\mathcal{G}, c, d$ satisfy the assumptions of Theorem 5.1. Then

$$
b_{k} \geq\left(1+\frac{d}{d-1} 2 c\right)^{k} \text {. }
$$

Remark. The corollary yields a lower estimate for the growth rate of distance balls for tessellating plane graphs with upper curvature bound. A corresponding upper estimate for the growth rate in graphs with lower curvature bound would have implications for the bottom of the essential spectrum of the graph-theoretic Laplacian (see, e.g., [Fu]).

Before proving Theorem 5.1 we introduce two lemmata. Let

$$
V_{k}^{2}=\left\{\left.v \in \partial B_{k}|| v\right|^{\mathrm{e}}=2\right\} \quad \text { and } \quad v_{k}^{2}=\left|V_{k}^{2}\right| .
$$

\section{Lemma 5.3.}

$$
\sum_{f \in A_{k+1}} E_{\partial f}=E_{\partial B_{k+1}}+E_{\partial B_{k}}+2 v_{k}^{2}
$$

Proof. Let $e \in \partial f$ be an edge of a face $f \in A_{k+1}$. Then either $e \in \partial B_{k+1}$ or $e \in \partial B_{k}$ or $e$ is a common edge of $f$ and another face $\tilde{f} \in A_{k+1}$. In the latter case, it follows from Theorem 3.2 that at least one vertex $v \in \partial e$ belongs to $\partial B_{k}$. It is clear that $|v|^{\mathrm{e}}=2$. If both vertices of $e$ belong to $\partial B_{k}$, then, by Proposition 3.5, we would have $\partial f-e \subset \partial B_{k}$, and thus $f \in C\left(f_{0}\right)$. So precisely one vertex $v \in \partial e$ belongs to $V_{k}^{2}$.

\section{Lemma 5.4 .}

$$
\sum_{f \in A_{k+1}} \sum_{v \in \partial f} \frac{1}{|v|} \leq \sum_{v \in \partial B_{k+1}} \frac{|v|^{\mathrm{i}}}{|v|}+\sum_{v \in \partial B_{k}} \frac{|v|^{\mathrm{i}}}{|v|}+v_{k}^{2}-\frac{a_{k+1}}{d}
$$

with $a_{k+1} / d=0$ if $d=\infty$.

Proof. In what follows we stick to the convention that, if a sum is taken over the boundary of a ball, the inner and exterior degrees of vertices are always with respect to this ball. We further define $a_{k+1}(v)=\left|\left\{f \in A_{k+1} \mid v \in \partial f\right\}\right|$.

From Proposition 3.4 it follows that every $v \in \partial B_{k+1}$ belongs to at most two faces of $A_{k+1}$. Thus we always have $a_{k+1}(v) \leq 2$. If $v \in \partial B_{k+1}$ belongs to two faces of $A_{k+1}$ (this is precisely the case if $a_{k+1}(v)=2$ ), then either $|v|_{B_{k+1}}^{\mathrm{i}}=2$ or $v \in \partial B_{k+1} \cap \partial B_{k}$. Correspondingly, if $v \in \partial B_{k}$ satisfies $a_{k+1}(v)=2$, we have either $|v|_{B_{k}}^{\mathrm{e}}=2$ or $v \in$ 
$\partial B_{k+1} \cap \partial B_{k}$. In particular, we conclude that $v \in \partial B_{k} \cap \partial B_{k+1}$ iff $v \in \partial B_{k}$ and $|v|_{B_{k}}^{\mathrm{e}}>2$. We remark further that, for every $f \in A_{k+1}$, all vertices $v \in \partial f$ are contained in $\partial B_{k} \cup \partial B_{k+1}$. Using these facts we deduce

$$
\begin{aligned}
& \sum_{f \in A_{k+1}} \sum_{v \in \partial f} \frac{1}{|v|}=\sum_{v \in \partial B_{k} \cup \partial B_{k+1}} \frac{a_{k+1}(v)}{|v|} \\
& =\sum_{\substack{v \in \partial B_{k+1} \\
|v|^{i}=1}} \frac{1}{|v|}+\sum_{\substack{\left.v \in \partial B_{k+1} \\
|v|\right|^{i}=2}} \frac{2}{|v|}+\sum_{\substack{v \in \partial B_{k} \\
|v|^{i}>2}} \frac{2}{|v|}+\sum_{\substack{v \in \partial B_{k} \\
|v|^{2}=1}} \frac{1}{|v|}+\sum_{\substack{v \in \partial B_{k} \\
|v|^{3}=2}} \frac{2}{|v|} \\
& \leq \sum_{v \in \partial B_{k+1}} \frac{|v|^{\mathrm{i}}}{|v|}+\sum_{\substack{v \in \partial B_{k} \\
|v|^{\mathrm{e}}=1}} \frac{1}{|v|}-\left(\sum_{\substack{v \in \partial B_{k+1} \\
|v|^{\mid}>2}}+\sum_{\substack{\left.v \in \partial B_{k} \\
|v|\right|^{\mathrm{e}}=2}}\right) \frac{1}{|v|}+\sum_{\substack{v \in \partial B_{k} \\
|v|^{\mathrm{e}}=2}} \frac{3}{|v|} \text {. }
\end{aligned}
$$

The inequality of the lemma follows from

$$
-\left(\sum_{\substack{v \in \partial B_{k} \\|v|^{i}>2}}+\sum_{\substack{\left.v \in \partial B_{k} \\|v|\right|^{2}=2}}\right) \frac{1}{|v|}+\sum_{\substack{v \in \partial B_{k} \\|v|^{2}=2}} \frac{3}{|v|} \leq-\frac{a_{k+1}}{d}+v_{k}^{2} .
$$

We remark that, in this last inequality, the estimate for the first term is obtained by using the following observation: Theorem 3.2 implies that any two subsequent faces $f_{1}, f_{2} \in A_{k+1}$ meet either in a vertex $v \in \partial B_{k+1}$ with $|v|_{B_{k+1}}^{\mathrm{i}}>2$ or along a common edge. In the latter case the common edge has a unique vertex $v^{\prime} \in \partial B_{k}$ with $\left|v^{\prime}\right|_{B_{k}}^{\mathrm{e}}=2$.

Proof of Theorem 5.1. We have

$$
a_{k+1}=\sum_{f \in A_{k+1}} \chi(f)+\frac{1}{2} \sum_{f \in A_{k+1}} E_{\partial f}-\sum_{f \in A_{k+1}} \sum_{v \in \partial f} \frac{1}{|v|} .
$$

It follows from Lemma 5.3 and formula (4) that

$$
\begin{aligned}
a_{k+1}= & \sum_{f \in A_{k+1}} \chi(f)+\frac{1}{2} E_{\partial B_{k+1}}+\frac{1}{2} E_{\partial B_{k}}+v_{k}^{2}-\sum_{f \in A_{k+1}} \sum_{v \in \partial f} \frac{1}{|v|} \\
= & \sum_{f \in A_{k+1}} \chi(f)-\chi\left(B_{k+1}\right)-\chi\left(B_{k}\right)+2+\sum_{v \in \partial B_{k+1}} \frac{|v|^{\mathrm{i}}}{|v|}+\sum_{v \in \partial B_{k}} \frac{|v|^{\mathrm{i}}}{|v|} \\
& +v_{k}^{2}-\sum_{f \in A_{k+1}} \sum_{v \in \partial f} \frac{1}{|v|} .
\end{aligned}
$$

Using Lemma 5.4 we get

$$
\begin{aligned}
\frac{d-1}{d} a_{k+1} & \geq \sum_{f \in A_{k+1}} \chi(f)-\chi\left(B_{k+1}\right)-\chi\left(B_{k}\right)+2 \\
& =-2 \chi\left(B_{k}\right)+2 \geq 2 c b_{k}+2 .
\end{aligned}
$$


Remark. There is also an asymptotic version of Corollary 5.2. It can be best stated in terms of the mean Euler-characteristic which, for an arbitrary polygon $P$, is defined as $\bar{\chi}(P)=\chi(P) /|P|$. Now, let $c>0$ be a constant satisfying $\lim \sup _{k \rightarrow \infty} \bar{\chi}\left(B_{k}\right)<-c$. Then the asymptotic version asserts that there are constants $a, k_{0}>0$ with

$$
b_{k} \geq a\left(1+2 \frac{d}{d-1} c\right)^{k} \quad \text { for all } \quad k \geq k_{0} .
$$

This follows immediately from the key estimate

$$
a_{k+1} \geq-2 \frac{d}{d-1}\left(\chi\left(B_{k}\right)-1\right)
$$

in the above proof.

\section{Regular Tessellating Graphs}

In this section we restrict our considerations to $n$-regular graphs, i.e., plane tessellating graphs $\mathcal{G}$ satisfying $|v|=n$, for all vertices $v \in \mathcal{V}$. For these graphs it is possible to derive growth formulas which are more precise than the general isoperimetric inequality of the previous section. Note also that non-positively curved $n$-regular graphs are always of type $(p, q)$.

Let $f_{0} \in \mathcal{F}$ be a fixed face in an $n$-regular graph $\mathcal{G}$. The numbers $a_{k}, b_{k}$ are defined as before. Moreover, we introduce the following numbers for any distance ball $B_{k}\left(f_{0}\right)$ :

$$
c_{k}^{j}=\left|\left\{\left.v \in \partial B_{k}|| v\right|^{\mathrm{e}}=j\right\}\right| .
$$

Lemma 6.1. Let $\mathcal{G}$ an n-regular graph with $C\left(f_{0}\right)=\emptyset$. Then

$$
\chi\left(B_{k}\right)=1+\sum_{j=1}^{n-1} \frac{n-2 j}{2 n} c_{k}^{j} .
$$

Proof. This is an immediate consequence of $E_{\partial B_{k}}=\sum_{j=1}^{n-1} c_{k}^{j}$ and (4).

Lemma 6.2. Let $\mathcal{G}$ be an n-regular graph with $C\left(f_{0}\right)=\emptyset$. Then the coefficients $c_{k}^{j}$ satisfy the following recurrence relations:

(i) $c_{k+1}^{n-2}=c_{k}^{2}$,

(ii) $c_{k+1}^{l-2}=c_{k}^{l}$, for $3 \leq l \leq n-1$.

Moreover, for $k \geq 0$,

$$
a_{k+2}-a_{k+1}=c_{k+1}^{n-1}-c_{k+1}^{1}
$$

Proof. We use the enumeration of the faces in $A_{k+1}$ introduced in Theorem 3.2. Note that any vertex $v \in \partial B_{k+1}$ with $|v|^{\mathrm{e}}=n-2$ belongs to precisely one common edge $e$ of 
two subsequent faces in $A_{k+1}$, and vice versa. The other vertex $v^{\prime}$ of this edge $e$ belongs to $\partial B_{k}$, and satisfies $|v|^{\mathrm{e}}=2$. Conversely, any vertex $v^{\prime} \in \partial B_{k}$ with $\left|v^{\prime}\right|^{\mathrm{e}}=2$ belongs to a common edge $e$ of two subsequent faces in $A_{k+1}$. This proves (i).

Note, for the proof of (ii), that any vertex $v \in \partial B_{k}$ with $|v|^{\mathrm{e}}=l, 3 \leq l \leq n-1$, is also a vertex of $\partial B_{k+1}$ with $|v|^{\mathrm{e}}=l-2$, and vice versa.

A moment's thought shows that $a_{k+1}$ coincides with the number of vertices $v \in \partial B_{k}$ with $|v|^{\mathrm{e}}>1$. Using this and the recurrence relations, we obtain

$$
\begin{aligned}
a_{k+1} & =\sum_{j=2}^{n-1} c_{k}^{j}=c_{k}^{2}+\sum_{j=3}^{n-1} c_{k}^{j} \\
& =c_{k+1}^{n-2}+\sum_{j=1}^{n-3} c_{k+1}^{j}=c_{k+1}^{1}+\sum_{j=2}^{n-1} c_{k+1}^{j}-c_{k+1}^{n-1} \\
& =c_{k+1}^{1}+a_{k+2}-c_{k+1}^{n-1} .
\end{aligned}
$$

Proposition 6.3. Let $\mathcal{G}$ be an n-regular graph without cut locus. Then we have, for $k \geq 1$,

$$
\chi\left(B_{k}\right)=1-\frac{n-2}{2 n}\left(a_{k+1}-a_{k}\right)+\sum_{j=2}^{n-2} \frac{n-2 j}{2 n} c_{k}^{j} .
$$

Proof. This follows immediately from the Lemmas 6.1 and 6.2.

Corollary 6.4. Let $\mathcal{G}$ be an n-regular graph without cut locus, and $n \in\{3,4,6\}$. Then, for $k \geq 1$,

$$
\chi\left(B_{k}\right)=1-\frac{n-2}{2 n}\left(a_{k+1}-a_{k}\right) .
$$

Proof. In the cases $n=3$ and $n=4$, there is nothing to do. Observe, for the case $n=6$, that $c_{k}^{j}=0$ for even values of $j$.

Remark. Interestingly, Corollary 6.4 has a counterpart in Riemannian geometry. The difference $a_{k+1}-a_{k}$ corresponds to a derivative in the continuous context, and an easy computation shows that

$$
\frac{1}{2 \pi} \int_{B_{r}(p)} \kappa(q) d \operatorname{vol}(q)=1-\frac{1}{2 \pi} L^{\prime}(r),
$$

where $B_{r}(p)$ denotes a geodesic ball of radius $r$ in a Riemannian 2-manifold $M, \kappa(q)$ denotes the Gaussian curvature, $r$ is assumed to be smaller than the injectivity radius of $p \in M$, and $L(r)$ denotes the length of the geodesic sphere $S_{r}(p)$.

Remark. The recurrence relations show, for $n$-regular graphs without cut locus, that the coefficients $\left\{c_{k+1}^{j}\right\}_{j}$ of the distance ball $B_{k+1}$ are already determined by the coefficients $\left\{c_{k}^{j}\right\}_{j}$ of the distance ball $B_{k}$, with the only exception of $c_{k+1}^{n-1}$. This implies, together 
with the other results in this section, that all three sequences $\left\{c_{k}^{j}\right\}_{j k},\left\{\chi\left(B_{k}\right)\right\}_{k}$ and $\left\{a_{k}\right\}_{k}$ determine each other mutually, and, consequently, carry the same information about the regular graph $\mathcal{G}$.

We, finally, compare the estimate (11) of the previous section with the statement of Corollary 6.4 in the particular case of an $n$-regular graph, $n \in\{3,4,6\}$. Estimate (11) yields in this case

$$
\chi\left(B_{k}\right) \geq 1-\frac{n-1}{2 n} a_{k+1},
$$

whereas Corollary 6.4 shows that

$$
\chi\left(B_{k}\right)=1-\frac{n-2}{2 n} a_{k+1}+\frac{n-2}{2 n} a_{k} .
$$

\section{Acknowledgements}

We thank Harm Derksen for helpful discussions, related to formula (4), in June 1997, at the Institute for Mathematics at Basel University. The first author thanks Prof. Hanspeter Kraft for the invitation to Basel and financial support. We also thank Jozef Dodziuk for his encouragement during the final preparation of the article in summer 1999, and the referee(s) for their numerous and valuable comments.

\section{References}

[BB] W. Ballmann, S. Buyalo, Nonpositively curved metrics on 2-polyhedra, Math. Z. 222 (1996), 97-134.

[Bo] P. L. Bowers, Negatively curved graph and planar metrics with applications to type, Michigan Math. J. 45 (1998), 31-53.

[DH] A. Dress, D. Huson, On tilings of the plane, Geom. Dedicata 24 (1987), 295-310.

[Do] J. Dodziuk, Difference equations, isoperimetric inequalities and transience of certain random walks, Trans. Amer. Math. Soc. 284 (1984), 787-794.

[Fu] K. Fujiwara, Growth and the spectrum of the Laplacian of an infinite graph, Tôhoku Math. J. 48 (1996), 293-302.

[GHV] E. Ghys, A. Haefliger, A. Verjovsky, Group Theory from a Geometrical Viewpoint, World Scientific, Singapore, 1991.

[GP] V. W. Guillemin, A. Pollack, Differentiable Topology, Prentice-Hall, Englewood Cliffs, NJ, 1974.

[GS] B. Grünbaum, G. Shephard, Tilings and Patterns, Freeman, New York, 1987.

[Li] P. Li, Complete surfaces of at most quadratic area growth, Comment. Math. Helv. 72 (1997), 67-71.

[Ly] R. C. Lyndon, On Dehn's algorithm, Math. Ann. 166 (1966), 208-228.

[Pó] G. Pólya, An elementary analogue to the Gauß-Bonnet theorem, Amer. Math. Monthly 61 (1954), 601-603.

[Ro] S. Rogmann, Wachstumsfunktionen von Pflasterungen, Diplomarbeit, Heinrich-Heine-Universität Düsseldorf, 1997.

[So] P. M. Soardi, Recurrence and transience of the edge graph of a tiling of the Euclidean plane, Math. Ann. 287 (1990), 613-626.

[Tho] C. Thomassen, Planarity and duality of finite and infinite graphs, J. Combin. Theory Ser. B 29 (1980), 244-271.

[Thu] W. P. Thurston, Shapes of polyhedra and triangulations of the sphere, in I. Rivin et al. (eds.): The Epstein Birthday Schrift, Geom. Topol. Monogr. 1, Warwick, Coventry, 1998, pp. 511-549. 
[Wh] A. T. White, Graphs, Groups and Surfaces, revised edition, Mathematics Studies 8, North-Holland, Amsterdam, 1984.

[Wo] W. Woess, A note on tilings and strong isoperimetric inequality, Math. Proc. Cambridge Philos. Soc. 124 (1998), 385-393.

[Yo] J. W. T. Youngs, Minimal embeddings and the genus of a graph, J. Math. Mech. 12 (1963), 303-315.

$[\dot{Z} u]$ A. Żuk, On the norms of the random walks on planar graphs, Ann. Inst. Fourier 47 (1997), 1463-1490.

Received September 27, 1999, and in revised form May 3, 2000. Online publication September 22, 2000. 